\title{
Computer simulations of the kinetics of irreversible enzyme inhibition by an unstable inhibitor
}

\author{
Christopher M. TOPHAM* \\ Department of Biochemistry, University of Oxford, South Parks Road, Oxford OX1 3QU, U.K., and Department of \\ Biochemistry, Trinity College, University of Dublin, Dublin 2, Ireland
}

\begin{abstract}
Computer simulations of the irrversible inhibition of an enzyme by an unstable inhibitor are presented. Data obtained at the end point of reaction are shown to conform poorly in many situations with relationships derived from integrated rate equations by setting $t=\infty$, and the implications concerning the experimental use of this method to determine kinetic constants describing inactivation are considered. The alternative approach of conducting experiments under conditions of inhibitor excess over enzyme is further discussed, and a graphical procedure is suggested for the description of time courses of reaction of enzyme with unstable inhibitor when an enzyme-inhibitor adsorptive complex is involved.
\end{abstract}

\section{INTRODUCTION}

Selective chemical modification of amino acid residues is a common method for gaining an understanding of the mechanisms of enzyme catalysis. Kinetic analysis of enzyme inactivation and modification by irreversible inhibitors is, however, often complicated by the use of reagents that are unstable in aqueous solution.

Most studies of irreversible enzyme inhibition are interpreted in terms of one of two basic mechanisms, depending on whether reaction between the enzyme (E) and inhibitor (I) to form an inactive species $\left(E^{*}\right)$ is considered to be a simple bimolecular reaction (eqn. 1) or to proceed through the intermediacy of an enzymeinhibitor adsorptive complex (EI) (eqn. 2):

$$
\begin{gathered}
\mathrm{E}+\mathrm{I} \stackrel{k_{i}}{\rightarrow} \mathrm{E}^{*} \\
\mathrm{E}+\mathrm{I} \underset{k_{-1}}{\stackrel{k_{+1}}{\longrightarrow}} \mathrm{EI} \stackrel{k_{+2}}{\rightarrow} \mathrm{E}^{*}
\end{gathered}
$$

Equations describing the time-dependent losses of enzyme activity by these mechanisms for the case of an inhibitor that is itself hydrolysed in a first-order reaction are well known.

For Mechanism 1 (eqn. 1):

$$
\ln a=\frac{k_{i}}{k^{\prime}}[\mathrm{I}]_{0}\left(\mathrm{e}^{-k^{\prime} t}-1\right)
$$

where $a$ is the fractional remaining activity at time $t,[\mathrm{I}]_{0}$ is the initial inhibitor concentration, and $k^{\prime}$ is the pseudo-first-order rate constant for the hydrolysis of the inhibitor (Purdie \& Heggie, 1970; Ashani et al., 1972; Rakitzis, 1974, 1984).

For Mechanism 2 (eqn. 2):

$$
\ln a=\frac{k_{+2}}{k^{\prime}} \cdot \ln \left(\frac{K_{i}+[\mathrm{I}]_{0} \mathrm{e}^{-k^{\prime} t}}{K_{i}+[\mathrm{I}]_{0}}\right)
$$

where $K_{i}=\left(k_{-1} / k_{+1}\right)$ is the dissociation constant of the enzyme-inhibitor complex (Purdie \& Heggie, 1970; Rakitzis, 1974).

One approach to the determination of the kinetic constants describing these mechanisms has been to incubate the enzyme with inhibitor for times long enough to allow the complete disappearance of the inhibitor before measurements of activity are made (Purdie \& Heggie, 1970; Ashani et al., 1972; Rakitzis, 1974, 1978, 1981, 1984). Accordingly, setting $t=\infty$ and rearranging eqn. (3) simplifies to give:

$$
\ln (1 / a)=\frac{k_{i}}{k^{\prime}}[\mathrm{I}]_{0}
$$

and $k_{i} / k^{\prime}$ is obtained as the slope of a plot of $\ln (1 / a)$ versus [I] (Ashani et al., 1972; Rakitzis, 1974). The corresponding equation formulated by Rakitzis (1974, 1984) for Mechanism 2 when the further condition $[\mathrm{I}]_{0} \gg K_{i}$ holds is:

$$
\log (1 / a)=\frac{k_{+2}}{k^{\prime}} \cdot \log [\mathrm{I}]_{0}-\frac{k_{+2}}{k^{\prime}} \cdot \log K_{i}
$$

The slope of a plot of $\log (1 / a)$ versus $\log [\mathrm{I}]_{0}$ should therefore be equal to $k_{+2} / k^{\prime}$ and the intercept on the $\log [\mathrm{I}]_{0}$ axis equal to $\log K_{i}$.

However, this approach, at least as applied to Mechanism 1, has been criticized recently on the grounds that over prolonged reaction periods a significant proportion of the inhibitor will eventually be consumed in the reaction with the enzyme, thus invalidating the assumption that the concentration of the inhibitor at any given moment is governed solely by its rate of hydrolysis in solution, a necessary condition for the derivation of eqns. (3) and (4) (Topham, 1985). In reply, Rakitzis (1985) has claimed that, since enzyme inactivation is a function of inhibitor concentration, it may safely be presumed that, in the period obtaining near the end of the reaction, the proportion of enzyme inactivation will be small. In order to test this claim, reactions of enzyme with stable inhibitor have been simulated for both mechanisms by using a computer, and the data have been analysed as described above. The relative merits of this method and a second approach in which experiments are conducted under conditions such that the inhibitor is present in excess over the entire observation period are further discussed, and a graphical procedure is suggested

* Present address: Department of Biochemistry, Trinity College, University of Dublin, Dublin 2, Ireland.

Vol. 240 


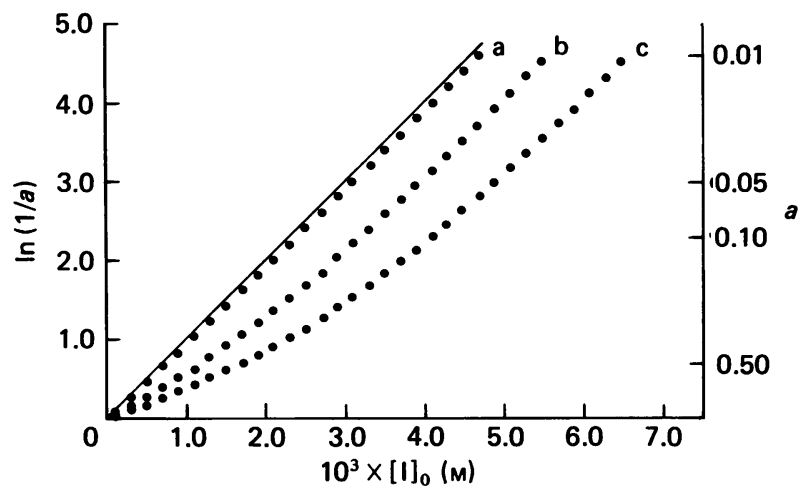

Fig. 1. Effect of alteration of total enzyme concentration on plots of simulated data obtained at the end point of reaction of enzyme with unstable inhibitor conforming to Mechanism 1 (eqn. 1)

The rate constants used were: $k_{i}=10.0 \mathrm{M}^{-1} \cdot \mathrm{s}^{-1}$; $k^{\prime}=1.0 \times 10^{-4} \mathrm{~s}^{-1}$. Total concentrations of enzyme were: curve a, $1.0 \times 10^{-6} \mathrm{M}$; curve b, $1.0 \times 10^{-5} \mathrm{M}$; curve $\mathrm{c}$, $2.0 \times 10^{-5} \mathrm{M}$. The continuous line was drawn according to eqn. (5).

for the description of time courses of reactions of enzyme with unstable inhibitor conforming to Mechanism 2.

\section{METHODS}

There is no analytical solution to the non-linear differential equations that describe the reaction of unstable inhibitor with enzyme for either Mechanism 1 or 2 . Solutions were computed for selected sets of values for the rate constants and total reactant concentrations by means of a variable-order variable-step formulation of the method of Gear (see Hall \& Watt, 1976) for stiff systems of differential equations, supplied as a subroutine D02EBF by the Numerical Algorithms Group, Oxford, U.K., and incorporated into a FORTRAN 1 V program. This subroutine was used previously by Fatania et al. (1982) in their studies of the displacement of NADPH from its complex with enzyme by NADP ${ }^{+}$. The program gives values for the concentrations of all the species after 25 equal time intervals. Time courses were simulated from zero time to either $7 \ln 2 / k^{\prime}$ (i.e. 7 half-times for the hydrolysis reaction) or until the inhibitor concentration (bound plus free in the case of Mechanism 2) had decayed to less than $1 \%$ of the initial enzyme concentration, whichever was the greater, at which point the reaction was considered to be complete. A PDP 11/03 computer (Digital Equipment Co.) was used.

\section{RESULTS}

\section{Computer simulations: Mechanism 1}

Reactions of enzyme with various initial concentrations of unstable inhibitor $\left([\mathrm{I}]_{0}\right)$ were simulated for long enough to allow the complete disappearance of the inhibitor, and the data were plotted according to eqn. (5). Results obtained with fixed values of $k_{i}\left(10.0 \mathrm{M}^{-1} \cdot \mathrm{s}^{-1}\right)$ and $k^{\prime}\left(1.0 \times 10^{-4} \mathrm{~s}^{-1}\right)$ at three different total enzyme concentrations $\left([\mathrm{E}]_{0}\right)$ are shown in Fig. 1. Plots are clearly curvilinear, and deviations from eqn. (5) are most apparent at high values of $[\mathrm{E}]_{0}$. For fixed $[\mathrm{E}]_{0}$ values the

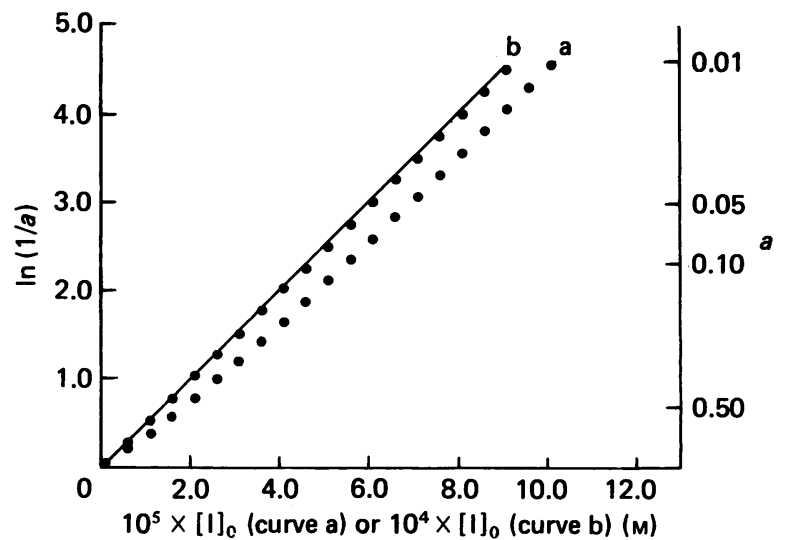

Fig. 2. Effect of variation in $k_{i} / \boldsymbol{k}^{\prime}$ on plots of simulated data obtained at the end point of reaction of enzyme with unstable inhibitor conforming to Mechanism 1 (eqn. 1)

The total concentration of enzyme used was $1.0 \times 10^{-5} \mathrm{M}$. The rate constants used were: curve a, $k_{i}=10.0 \mathrm{M}^{-1} \cdot \mathrm{s}^{-1}$; $k^{\prime}=2.0 \times 10^{-4} \mathrm{~s}^{-1} ; \quad$ curve b, $k_{i}=10.0 \mathrm{M}^{-1} \cdot \mathrm{s}^{-1}$; $k^{\prime}=2.0 \times 10^{-3} \mathrm{~s}^{-1}$. The continuous line was drawn according to eqn. (5).

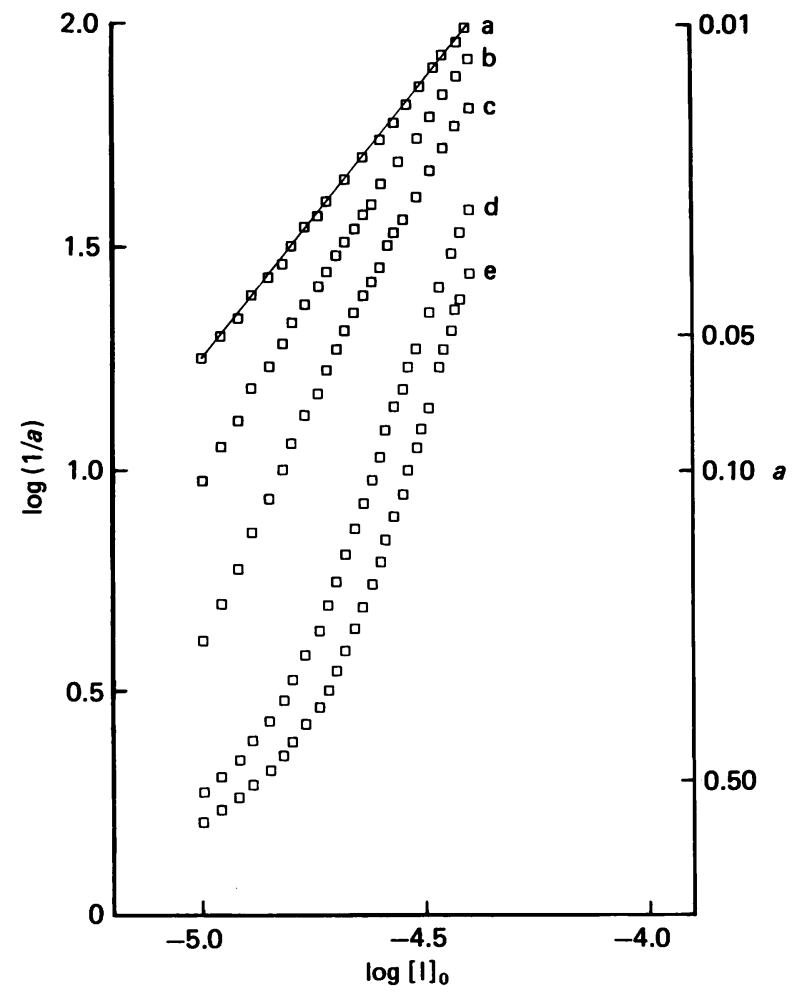

Fig. 3. Effect of alteration of total enzyme concentration on plots of simulated data obtained at the end point of reaction of enzyme with unstable inhibitor conforming to Mechanism 2 (eqn. 2)

The rate constants used were: $k_{+1}=1.0 \times 10^{7} \mathrm{M}^{-1} \cdot \mathrm{s}^{-1}$; $k_{-1}=10.0 \mathrm{~s}^{-1} ; k_{+2}=5.0 \times 10^{-4} \mathrm{~s}^{-1} ; k^{\prime}=4.0 \times 10^{-4} \mathrm{~s}^{-1}$. Total concentrations of enzyme were: curve $a$, $1.0 \times 10^{-6} \mathrm{M}$; curve b, $5.0 \times 10^{-6} \mathrm{M}$; curve c, $1.0 \times 10^{-5} \mathrm{M}$; curve d, $2.0 \times 10^{-5} \mathrm{M} ;$ curve e, $2.5 \times 10^{-5} \mathrm{M}$. The continuous line was drawn according to eqn. (6). 

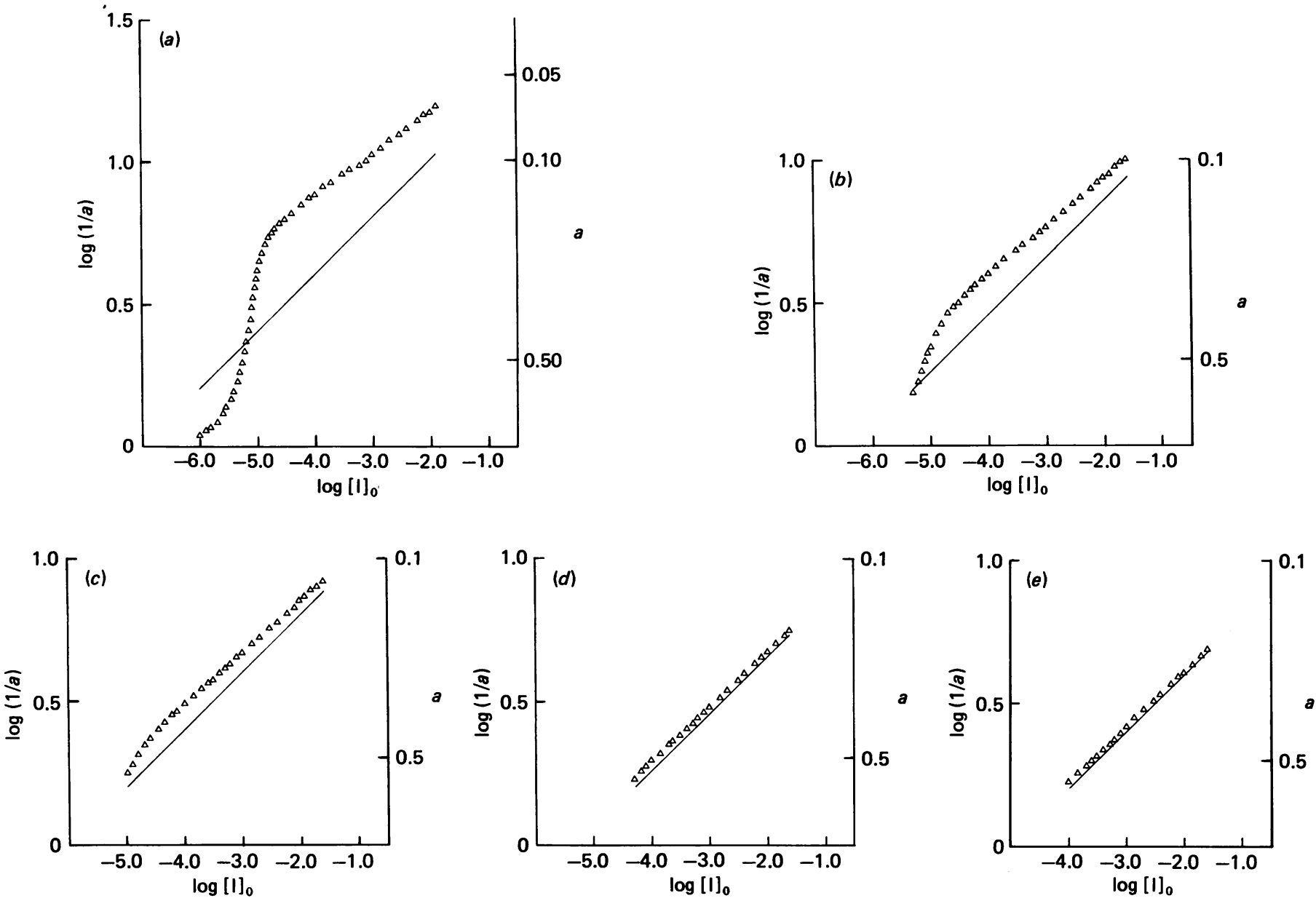

Fig. 4. Effect of variation in $K_{i}$ on plots of simulated data obtained at the end point of reaction of enzyme with unstable inhibitor conforming to Mechanism 2 (eqn. 2)

The rate constants used were: $k_{+1}=1.0 \times 10^{7} \mathrm{M}^{-1} \cdot \mathrm{s}^{-1} ; k_{+2}=5.0 \times 10^{-4} \mathrm{~s}^{-1} ; k^{\prime}=2.5 \times 10^{-3} \mathrm{~s}^{-1} ;(a) k_{-1}=1.0 \mathrm{~s}^{-1} ;(b)$ $k_{-1}=5.0 \mathrm{~s}^{-1} ;(c) k_{-1}=10.0 \mathrm{~s}^{-1} ;(d) k_{-1}=50.0 \mathrm{~s}^{-1} ;(e) k_{-1}=100.0 \mathrm{~s}^{-1}$. The total concentration of enzyme was $1.0 \times 10^{-5} \mathrm{M}^{-1}$. Continuous lines were drawn according to eqn. (6).

relative (but not the absolute) differences between simulated $\ln (1 / a)$ values and those predicted by eqn. (5) become smaller as $[\mathrm{I}]_{0}$ is increased. These observations are consistent with the consumption of a significant proportion of the inhibitor in the reaction with the enzyme and lead to an underestimation of the fractional residual activity for a given value of $[I]_{0}$. Fig. 2 shows the effect of increasing $k^{\prime}$ relative to $k_{i}$ at a fixed $[\mathrm{E}]_{0}$. Agreement with eqn. (5) is better for small values of $k_{i} / k^{\prime}$, partly as a result of the necessity to use larger values of $[\mathrm{I}]_{0}$ to obtain the same degree of inhibition, but it is also apparent that the relative differences in $\ln (1 / a)$ at fixed $[\mathrm{I}]_{0}$ values become smaller as $k_{i} / k^{\prime}$ decreases (see also curve $b$ in Fig. 1), and this is due to a shift in the reaction flux with respect to inhibitor towards that predicted by a simple pseudo-first-order decomposition process.

\section{Computer simulations: Mechanism 2}

Analogous simulations were performed for Mechanism 2, and the data were plotted according to eqn. (6). In each case $k_{+1}$ was assigned a value of $1.0 \times 10^{7} \mathrm{M}^{-1} \cdot \mathrm{s}^{-1}$ and $k_{+2} \ll k_{-1}$, a sufficient condition for the existence of a quasi-equilibrium between EI, E and I over the entire reaction course (see Cornish-Bowden, 1979; Brockle- hurst, 1979). The smallest values of $[\mathrm{I}]_{0}$ were set equal to $10 K_{i}$.

As in the case of Mechanism 1, deviations from eqn. (6) are greatest with high values of $[E]_{0}$ with fixed values for the four rate constants $\left(k_{+2} / k^{\prime}=1.25\right.$; $\left.K_{i}=1.0 \times 10^{-6} \mathrm{M}\right)$ (Fig. 3), and better agreement is obtained as $[\mathrm{I}]_{0}$ is increased for each curve. It will be noted that in the general case plots of $\log (1 / a)$ versus $\log [\mathrm{I}]_{0}$ are sigmoid curves. Fig. 4 shows the effect of changes in $K_{i}$ at fixed values of $k_{+2} / k^{\prime}(0.2)$ and [E] $\left(1.0 \times 10^{-5} \mathrm{M}\right)$, and it can be seen that the larger $K_{i}$ becomes the closer the approximation of the simulated curve to that predicted by eqn. (6). This is to be expected, since larger values of $[I]_{0}$ are required to attain the same level of inhibition, and for a given [I] $]_{0}$ value a smaller proportion of the inhibitor will exist as EI (and $E^{*}$ ) over the time course of reaction. A further notable feature of the curves shown in Fig. 4 is that greater inhibition can be obtained than is predicted by eqn. (6). This arises as a result of the existence of elevated concentrations of $I$ and EI over much of the period before the completion of the reaction compared with those expected for the hydrolysis of an inhibitor occurring in a straightforward pseudo-first-order manner. This phenomenon is favoured 
by a decrease in $k_{+2} / k^{\prime}$ at a particular [I] value (cf. curve $c$ of Fig. 3 and Fig. $4 c$ ). The use of larger $[\mathrm{I}]_{0}$ values diminishes the effect in all circumstances.

\section{DISCUSSION}

The computer simulation studies show that data obtained at the end point of reaction of an unstable irreversible inhibitor with enzyme conform poorly in many situations with relationships derived from integrated rate equations by setting $t=\infty$. Plots of the data according to eqns. (5) and (6) were shown to be curvilinear, and in the general case for Mechanism 2 sigmoid. These deviations from linearity cannot be attributed to the definition of the end point of reaction used here, since agreement with eqns. (5) and (6) was best at high extents of inhibition where any error would be most manifest, and are actually the result of a logical contradiction in the formulation of these relationships (Topham, 1985). Deviations from linearity of the type shown in Figs. 1 and 2 have been observed experimentally by Makoff \& Malcolm (1981) in their studies of the modification of denatured aldolase by the water-unstable compound methyl acetimidate, but were attributed to a change in $\mathrm{pH}$ at high reagent concentrations.

It is clear from this study that attempts to determine kinetic parameters describing inactivation from apparently linear portions of experimental curves could, in several cases, generate erroneous values. Use of this method could also lead one to conclude that inactivation was in some way enzyme-concentration-dependent (see Figs. 1 and 3), and genuine enzyme-concentrationdependency would be difficult to prove. There are, however, circumstances in which eqns. (5) and (6) are reasonable approximations, but it would still be essential to show experimentally that inactivation could be described by the same kinetic constants at much lower (or higher) enzyme concentrations for the same range of initial inhibitor concentrations. In addition, linearity should be established over a wide range of extents of inactivation. This may not be possible in the case of Mechanism 2 when the condition $[\mathrm{I}]_{0} \gg K_{i}$ (Rakitzis, 1974,1984 ) is rigorously imposed (see for example curve a in Fig. 3).

Many of these problems may be circumvented by using the information that can be gained from a study of the time course of inactivation under conditions of inhibitor excess over enzyme concentration and an independent knowledge of the value of the rate constant for the hydrolysis of the inhibitor. This is achieved in the case of Mechanism 1 by plotting enzyme inactivation data according to eqn. (7), a rearrangement of eqn. (3) (Topham, 1985):

$$
\ln a=-k_{i}[\mathrm{I}]_{0} t^{\prime}
$$

where $t^{\prime}$ is used to denote $\left(1-\mathrm{e}^{-k^{\prime} t}\right) / k^{\prime}$, which has the dimensions of time. Plots of $\ln a$ versus $t^{\prime}$ have been successfully used to analyse the kinetics of inactivation of rat liver $S$-adenosylhomocysteinase (Gomi \& Fujioka, 1983), ribulose-1,5-bisphosphate carboxylase/oxygenase (Paech, 1985) and sheep liver 6-phosphogluconate dehydrogenase (Topham \& Dalziel, 1986) by diethyl pyrocarbonate. Paquatte \& Tu (1986), apparently unaware of previous work in this area, have proposed the use of a semi-logarithmic plot of fractional residual enzyme activity versus $\left(1-\mathrm{e}^{-k^{\prime} t}\right)$ and have determined second-order rate constants for the inactivation of salicylate hydroxylase and bacterial luciferase by diethyl pyrocarbonate.

In the case of Mechanism 2, the graphical procedure outlined below, through careful choice of inhibitor concentration, enables $k_{+2}$ to be determined independently of $K_{i}$, and then utilizes this value to estimate $K_{i}$. Thus, when $[\mathrm{I}]_{0} \mathrm{e}^{-k^{\prime} t} \gg K_{i}$, and hence when $[\mathrm{I}]_{0} \gg K_{i}$, eqn. (4) simplifies to give (Purdie \& Heggie, 1970):

$$
\ln a=-k_{+2} t
$$

and so $k_{+2}$ can be obtained as the negative slope of a plot of $\ln a$ versus time. It should be noted that the condition $[\mathrm{I}]_{0} \gg K_{i}$ is also required for the formulation of eqn. (6) (Rakitzis, 1974, 1978), and this, regardless of other considerations, is incompatible with the accurate determination of $K_{i}$, since it involves extrapolation over at least one order of magnitude. However, $K_{i}$ may be determined in a separate experiment in which the inhibitor is allowed to hydrolyse over a concentration range of comparable magnitude to $K_{i}$. A plot of $a^{k^{\prime} / k_{+2}}$ versus $[\mathrm{I}]_{0} \mathrm{e}^{-k^{\prime} t}$ (i.e. $\left.[\mathrm{I}]\right)$ is then constructed according to eqn. (9), a transformation of eqn. (4):

$$
a^{k^{\prime} / k_{+2}}=\frac{K_{i}}{K_{i}+[\mathrm{I}]_{0}}+\frac{1}{K_{i}+[\mathrm{I}]_{0}}[\mathrm{I}]_{0} \mathrm{e}^{-k^{\prime} t}
$$

and $K_{i}$ is obtained as the ratio of the intercept on the $a^{k^{\prime} / k_{+2}}$ axis to the slope. Inspection of eqn. (9) reveals that, if several $[\mathrm{I}]_{0}$ values are chosen, a series of straight lines will be obtained, all of which intersect the $[\mathrm{I}]_{0} \mathrm{e}^{-k^{\prime} t}$ axis at a value equal to $-K_{i}$, and so this may be used to confirm that the estimates of $k^{\prime}$ and $k_{+2}$ are correct.

I am grateful to Dr. K. Dalziel and Professor K. F. Tipton for helpful discussion and comments.

\section{REFERENCES}

Ashani, Y., Wins, P. \& Wilson, I. B. (1972) Biochim. Biophys. Acta 284, 427-434

Brocklehurst, K. (1979) Biochem. J. 181, 775-778

Cornish-Bowden, A. (1979) Eur. J. Biochem. 93, 383-385

Fatania, H. R., Matthews, B. \& Dalziel, K. (1982) Proc. R. Soc. London Ser. B 214, 369-387

Gomi, T. \& Fujioka, M. (1983) Biochemistry 22, 137-143

Hall, G. \& Watt, J. M. (eds.) (1976) Modern Numerical Methods for Ordinary Differential Equations, Clarendon Press, Oxford

Makoff, A. J. \& Malcolm, A. D. B. (1981) Biochem. J. 193, 245-249

Paech, C. (1985) Biochemistry 24, 3194-3199

Paquatte, O. \& Tu, S.-C. (1986) Biochim. Biophys. Acta 869, 359-362

Purdie, J. E. \& Heggie, R. M. (1970) Can. J. Biochem. 48, 244-250

Rakitzis, E. T. (1974) Biochem. J. 141, 601-603

Rakitzis, E. T. (1978) J. Theor. Biol. 75, 239-243

Rakitzis, E. T. (1981) Biochem. J. 199, 462-463

Rakitzis, E. T. (1984) Biochem. J. 217, 341-351

Rakitzis, E. T. (1985) Biochem. J. 227, 1026-1027

Topham, C. M. (1985) Biochem. J. 227, 1025-1026

Topham, C. M. \& Dalziel, K. (1986) Eur. J. Biochem. 155, 87-94 